\title{
Prediction of outcome in breast cancer patients using test parameters from complete blood count
}

\author{
PINGPING ZHANG ${ }^{1 *}$, YULONG ZONG ${ }^{1 *}$, MOHAN LIU $^{2 *}$, YANHONG TAI $^{3}$, YUAN CAO $^{1}$ and $\mathrm{CHENGIN} \mathrm{HU}^{1}$ \\ ${ }^{1}$ Department of Laboratory Medicine, General Hospital of Jinan Military Region, Jinan, Shandong 250031; \\ ${ }^{2}$ Department of Cardiology, Chinese PLA General Hospital, Beijing $100853 ;{ }^{3}$ Department of Pathology, \\ General Hospital of Jinan Military Region, Jinan, Shandong 250031, P.R. China
}

Received November 27, 2015; Accepted March 7, 2016

DOI: $10.3892 / \operatorname{mco} .2016 .827$

\begin{abstract}
The aim of this study was to evaluate the prognostic effect of test parameters from pretreatment complete blood count (CBC) for predicting outcome in breast cancer patients. A total of 162 patients with breast cancer and a long follow-up were enrolled in this study. Red cell indices (RCIs) and neutrophil-lymphocyte ratio (NLR) from CBC prior to treatment, as well as related clinical data, were retrospectively collected. We evaluated the association of RCI and NLR with tumor size, clinical stage, histological grade, estrogen receptor, progesterone receptor, and human epidermal growth factor receptor 2 status. We further performed survival analysis and Cox multivariate analysis, stratified by RCI and NLR median values, to evaluate their prognostic effects. In the disease-free survival (DFS) analysis, patients in the higher mean corpuscular hemoglobin $(\mathrm{MCH})$ and NLR groups exhibited shorter DFS times compared with those in the lower MCH and NLR groups $(\mathrm{P}=0.017$ for $\mathrm{MCH}$ and $\mathrm{P}=0.039$ for $\mathrm{NLR})$. The univariate analysis revealed that both $\mathrm{MCH}$ and NLR were significantly associated with DFS. The Cox multivariate analysis demonstrated that only $\mathrm{MCH}$ was an independent predictor associated with disease relapse (hazard ratio $=1.975$, 95\% confidence interval: $1.118-3.487, \mathrm{P}=0.019)$, whereas no
\end{abstract}

Correspondence to: Professor Chengjin Hu or Dr Yuan Cao, Department of Laboratory Medicine, General Hospital of Jinan Military Region, no. 25 Shifan Road, Tianqiao District, Jinan, Shandong 250031, P.R. China

E-mail: hcj6219@163.com

E-mail: cao.yuan@outlook.com

${ }^{*}$ Contributed equally

Abbreviations: $\mathrm{BMI}$, body mass index; $\mathrm{CBC}$, complete blood count; DFS, disease-free survival; HGB, hemoglobin; HCT, hematocrit; $\mathrm{MCV}$, mean corpuscular volume; $\mathrm{MCH}$, mean corpuscular hemoglobin; MCHC, mean corpuscular hemoglobin concentration; NLR, neutrophil-to-lymphocyte ratio; RBC, red blood cell; RCI, red cell indices; OS, overall survival

Key words: breast cancer, red cell indices, mean corpuscular hemoglobin, neutrophil-to-lymphocyte ratio, prognosis index was associated with overall survival. Our results suggest that $\mathrm{MCH}$ prior to treatment may be a predictive marker associated with DFS in breast cancer.

\section{Introduction}

Breast cancer, the most commonly diagnosed malignancy in women, is a heterogeneous disease encompassing phenotypically distinct tumor subtypes $(1,2)$. It is estimated that breast cancer is responsible for $>1,300,000$ cases and 450,000 deaths annually worldwide (3). In China, newly diagnosed cases and deaths account for 12.2 and $9.6 \%$ of global breast cancer patients, respectively $(4,5)$. With the advances in treatment and early detection, breast cancer mortality decreased by $34 \%$ from 1990 to 2010 (6). In America, the 5-year survival rate for breast cancer patients is $>75 \%$, while the 5-year survival rate for patients with breast cancer in Shanghai (the most industrialised city in China) was found to be $78 \%$ compared with 58\% reported in Qidong (a rural neighbouring area of Shanghai) (4). Therefore, there is a need for simple, reliable and non-invasive prognostic biomarkers to enable clinicians to perform risk evaluation in breast cancer patients prior to or during the treatment process.

Tumor markers in breast cancer have been investigated for nearly 20 years $(7,8)$. Recently, certain indices from complete blood count (CBC) appear to be useful for predicting outcomes in patients with breast cancer. The neutrophil-to-lymphocyte ratio (NLR) has been used not only as a biomarker of inflammation, but also as a prognostic index for various common solid tumors, such as gastric cancer, breast carcinoma, colorectal carcinoma, nasopharyngeal cancer and malignant melanoma (9). Azab et al first evaluated NLR in predicting short- and long-term mortality in breast cancer patients (10) and found that patients in the highest NLR quartile had higher 1- and 5-year mortality rates compared with those in the lowest quartile. Several studies reported similar results (11-16). In addition, low hemoglobin (HGB) level prior to each cycle of adjuvant polychemotherapy, as a marker of anemia, was reported to be associated with worse local relapse-free survival in patients with primary breast cancer (17-21). Furthermore, mean corpuscular volume (MCV) was also assessed during capecitabine therapy in metastatic breast cancer $(9,22)$. However, in the study of Bozkurt et al (23), no significant 
difference was identified between tumor response and increase in MCV. Moreover, a limited number of previous studies were based on Chinese patients with breast cancer. Thus far, the clinical utility of pretreatment $\mathrm{CBC}$ parameters in breast cancer prognosis requires further validation.

Consequently, in this retrospective analysis, we attempted to investigate the prognostic value of red cell indices (RCIs) and NLR in breast cancer patients with a long follow-up, taking into account several clinicopathological characteristics, such as tumor markers, clinical stage and histological grade.

\section{Patients and methods}

Patients and clinical data. We performed a retrospective review of patients undergoing complete resection of breast cancer between 2001 and 2005. A total of 162 breast cancer patients from the Chinese PLA General Hospital (Beijing, China) were enrolled in this study. The inclusion criteria were as follows: Patients diagnosed with breast cancer, with data on CBC, including RCIs and leukocyte differential count, prior to initiating any chemotherapy. The exclusion criteria were as follows: Patients without any blood count data prior to chemotherapy, presence of active infection, presence of coexisting hematological malignancies or other hematological disorders, autoimmune disorders, and patients on recent steroid therapy. These patients have been followed for up to 9.6 years. The median overall survival (OS) and disease-free survival (DFS) were 74.6 months (range, 2.4-115.6 months) and 69.9 months (range, 1.5-115.6 months), respectively. The design of this study was approved by our Hospital's Ethics Committee and all the patients provided written informed consent.

Data were collected from patient medical records, pathology reports, and blood results recorded on admission prior to the initiation of any treatment, including radiotherapy, chemotherapy, or surgery. The medical records for each patient were independently reviewed by two physicians for the baseline characteristics (Table I). All the test results were obtained using the same equipment. RCIs from blood test results were collected, including red blood cell (RBC) count, HGB, hematocrit (HCT), MCV, mean corpuscular hemoglobin $(\mathrm{MCH})$ and mean corpuscular hemoglobin concentration (MCHC). Neutrophil-to-lymphocyte ratio (NLR) was also calculated based on the CBC. Breast cancer patients were divided into two groups (high vs. low) according to the median value of each index. Accordingly, the two groups obtained from our data for NLR were NLR $<1.81$ and $\geq 1.81$. Similarly, the two groups for $\mathrm{MCH}$ were $\mathrm{MCH}<30.6$ and $\geq 30.6$. Table I summarizes the patient characteristics according to the $\mathrm{MCH}$ and NLR groups.

Statistical analysis. Statistical analysis was performed using SPSS software, version 21.0 (Armonk, NY, USA). Continuous variables are presented as mean \pm standard deviation and categorical variables are presented as frequencies and percentages. Student's t-test or Mann-Whitney U test was used to test the differences between the two groups. For group comparisons, Chi-square and Fisher's exact tests were used for categorical variables. Kaplan-Meier survival curves were plotted for DFS and OS in the different NLR and $\mathrm{MCH}$ groups. The statistical differences between these curves were detected using log-rank tests. Clinicopathological parameters are known to be associated with prognosis, including tumor size (T2-3 vs. T1), histological grade (III vs. I/II), estrogen receptor (ER) status (negative vs. positive), progesterone receptor (PR) status (negative vs. positive), human epidermal growth factor receptor 2 (HER2) status (negative vs. positive), and the value of each RCI separately (high vs. low) were tested with univariate analysis. Variables that were found to be significant in the univariate analysis were then entered in a stepwise multivariate Cox proportional hazard regression model to ascertain the individual contribution of factors associated with DFS and OS.

\section{Results}

Patient characteristics. Table I presents the general information and characteristics related to the tumors of the 162 breast cancer patients by $\mathrm{MCH}$ and NLR. The mean age of our population was 50.8 years. According to the American Joint Committee on Cancer staging guidelines, 110 patients were classified as stage I/II and 52 as stage III. All the patients were followed up until death or the last date of this study. The 10-year DFS and OS of all 162 breast cancer patients were 67 and $77 \%$, respectively. NLR was not found to be associated with age, histological grade, tumor size, lymph node status, ER status, PR status, or HER2 status in the population studied. As regards $\mathrm{MCH}$, the higher $\mathrm{MCH}$ group had significantly higher rates of HER2-positive tumors $(\mathrm{P}=0.044$; Table I).

Survival analysis of RCIs and NLR. We evaluated the correlation of RCIs and NLR with prognosis of breast cancer. Kaplan-Meier survival analysis and log-rank tests were performed using patients' postoperative survival. The Kaplan-Meier survival curves demonstrated that patients with high $\mathrm{MCH}$ exhibited worse DFS compared with those with low $\mathrm{MCH}(\mathrm{P}=0.017)$ (Fig. 1A), whereas higher $\mathrm{MCH}$ only showed a trend of higher patient mortality (Fig. 1B). Similarly, patients with high NLR had shorter DFS times compared with those with low NLR ( $\mathrm{P}=0.039)$ (Fig. 1C). However, patients with low NLR did not exhibit significantly longer OS times compared with those with high NLR (Fig. 1D). The values of the remaining five RCIs were not correlated with DFS or OS times.

Predictive value of $\mathrm{MCH}$. The univariate analysis revealed that tumor size, histological grade, clinical stage and lymph node status were significantly associated with DFS and OS in our cohort, whereas PR was significantly associated with OS (Table II). As regards RCIs, the univariate analysis revealed that only $\mathrm{MCH}$ was significantly associated with DFS, whereas no index was associated with OS (Table II). A Cox proportional hazards model generated a univariate hazard ratio (HR) of 1.959 [95\% confidence interval (CI): 1.114-3.445, $\mathrm{P}=0.020]$ for DFS by comparing high and low MCH. For OS, the univariate $\mathrm{HR}$ of high $\mathrm{MCH}$ compared with low $\mathrm{MCH}$ was 1.848 (95\% CI: $0.885-3.858, \mathrm{P}=0.102)$. To further investigate the effect of all the parameters showing significance in the univariate analysis regarding the prognosis of breast cancer patients, a multivariate Cox proportional hazards model was yielded for DFS using the following variables: $\mathrm{MCH}$, tumor 
Table I. Patient characteristics.

\begin{tabular}{|c|c|c|c|c|c|c|c|}
\hline \multirow[b]{2}{*}{ Characteristics } & \multirow[b]{2}{*}{ Overall } & \multicolumn{2}{|c|}{$\mathrm{MCH}$} & \multirow[b]{2}{*}{$\mathrm{P}$-value } & \multicolumn{2}{|c|}{ NLR } & \multirow[b]{2}{*}{ P-value } \\
\hline & & Low & High & & Low & High & \\
\hline Patients & $162(100 \%)$ & 83 & 79 & & 80 & 82 & \\
\hline Age \pm SD (years) & $50.8 \pm 10.6$ & $50.6 \pm 10.1$ & $50.9 \pm 11.1$ & 0.847 & $51.9 \pm 10.3$ & $49.9 \pm 10.8$ & 0.228 \\
\hline T stage, $\mathrm{n}$ & & & & 0.379 & & & 0.774 \\
\hline $\mathrm{T} 1$ & 53 & 26 & 27 & & 28 & 25 & \\
\hline $\mathrm{T} 2$ & 89 & 50 & 39 & & 44 & 45 & \\
\hline $\mathrm{T} 3$ & 17 & 6 & 11 & & 6 & 9 & \\
\hline $\mathrm{T} 4$ & 3 & 1 & 2 & & 2 & 1 & \\
\hline N stage, n (\%) & & & & 0.915 & & & 0.345 \\
\hline No & $73(45.1)$ & 36 & 37 & & 37 & 36 & \\
\hline $\mathrm{N} 1$ & $42(25.9)$ & 23 & 19 & & 23 & 19 & \\
\hline $\mathrm{N} 2$ & $22(13.6)$ & 12 & 10 & & 7 & 15 & \\
\hline N3 & $25(15.4)$ & 12 & 13 & & 13 & 12 & \\
\hline Histological grade, n (\%) & & & & 0.216 & & & 0.593 \\
\hline $\mathrm{I} / \mathrm{II}$ & $120(74.1)$ & 65 & 55 & & 61 & 59 & \\
\hline III & $42(25.9)$ & 18 & 24 & & 19 & 23 & \\
\hline Clinical stage, n (\%) & & & & 0.616 & & & 0.241 \\
\hline $\mathrm{I} / \mathrm{II}$ & $110(67.9)$ & 58 & 52 & & 58 & 52 & \\
\hline III & $52(32.1)$ & 25 & 27 & & 22 & 30 & \\
\hline ER, n $(\%)$ & & & & 0.552 & & & 0.156 \\
\hline Positive & $87(53.7)$ & 46 & 41 & & 38 & 49 & \\
\hline Negative & $74(45.7)$ & 37 & 37 & & 42 & 32 & \\
\hline Unkonwn & $1(0.6)$ & 0 & 1 & & 0 & 1 & \\
\hline PR, n $(\%)$ & & & & 0.888 & & & 0.156 \\
\hline Positive & $77(47.6)$ & 41 & 36 & & 32 & 45 & \\
\hline Negative & $83(51.2)$ & 41 & 42 & & 48 & 35 & \\
\hline Unkonwn & $2(1.2)$ & 1 & 1 & & 0 & 2 & \\
\hline HER 2, n (\%) & & & & 0.044 & & & 0.1406 \\
\hline Positive & $37(22.8)$ & 13 & 24 & & 22 & 15 & \\
\hline Negative & $124(76.5)$ & 70 & 54 & & 58 & 76 & \\
\hline Unkonwn & $1(0.6)$ & 0 & 1 & & 0 & 1 & \\
\hline
\end{tabular}

$\mathrm{MCH}$, mean corpuscular hemoglobin; NLR, neutrophil-to-lymphocyte ratio; ER, estrogen receptor; PR, progesterone receptor; HER2, human epidermal growth factor receptor 2; SD, standard deviation.

size, lymph node status, clinical stage and histological grade (Table III). Following backward elimination, the final model consisted of $\mathrm{MCH}$, tumor size and clinical stage. The multivariate $\mathrm{HR}$ of high $\mathrm{MCH}$ compared with low $\mathrm{MCH}$ was 1.975 (95\% CI: $1.118-3.487, \mathrm{P}=0.019)$, which demonstrated that $\mathrm{MCH}$ was an independent factor associated with DFS in these patients (Table IV).

Predictive value of NLR. A Cox proportional hazards model yielded a univariate $\mathrm{HR}=1.808$ (95\% CI: 1.021-3.202, $\mathrm{P}=0.042$ ) of high compared with low NLR for DFS, whereas NLR was not significantly associated with $\mathrm{OS}(\mathrm{P}=0.579)$ (Table III). A multivariate Cox proportional hazards model was constructed for DFS by incorporating the following variables: NLR, tumor size, lymph node status, clinical stage and histological grade. The multivariate HR of high compared with low NLR was 1.435 (95\% CI: 0.803-2.563, $\mathrm{P}=0.223$ ) (Table IV).

\section{Discussion}

In this study, we performed a systematic evaluation of the prognostic significance of RCIs as well as of NLR in breast cancer and revealed three major findings: i) $\mathrm{MCH}$ values were positively associated with HER2 status; ii) patients with higher $\mathrm{MCH}$ and NLR values prior to treatment were found to be associated with a poor prognosis; and iii) the multivariate Cox analysis demonstrated that $\mathrm{MCH}$ was an independent factor associated with DFS in our cohort, while NLR did not exhibit 
Table II. Univariate analysis for disease-free and overall survival of patients with breast cancer.

\begin{tabular}{|c|c|c|c|c|}
\hline \multirow[b]{2}{*}{ Factors } & \multicolumn{2}{|c|}{ Disease-free survival } & \multicolumn{2}{|c|}{ Overall survival } \\
\hline & Hazard ratio $(95 \% \mathrm{CI})$ & P-value & Hazard ratio $(95 \% \mathrm{CI})$ & P-value \\
\hline Age & $0.987(0.961-1.014)$ & 0.341 & $1.003(0.970-1.038)$ & 0.848 \\
\hline Tumor size, T2-T4 vs. T1 & 4.203 (1.792-9.857) & 0.001 & $7.243(1.725-30.412)$ & 0.007 \\
\hline Lymph node N1-3 vs. N0 & $3.424(1.756-6.679)$ & $<0.001$ & $5.973(2.083-17.130)$ & 0.001 \\
\hline Histology grade, III vs. I/II & $2.228(1.302-4.019)$ & 0.004 & $3.656(1.777-7.524)$ & $<0.001$ \\
\hline Clinical stage, III vs. I/II & $4.228(2.402-7.442)$ & $<0.001$ & $5.036(2.352-10.786)$ & $<0.001$ \\
\hline $\mathrm{ER}$ & $0.696(0.401-1.206)$ & 0.196 & $0.602(0.292-1.241)$ & 0.169 \\
\hline PR & $0.617(0.351-1.082)$ & 0.092 & $0.348(0.155-0.782)$ & 0.011 \\
\hline HER 2 & $1.607(0.879-2.940)$ & 0.124 & $1.760(0.800-3.873)$ & 0.160 \\
\hline $\mathrm{RBC}$ & $0.640(0.366-1.119)$ & 0.117 & $0.862(0.421-1.766)$ & 0.684 \\
\hline HGB & $0.929(0.536-1.608)$ & 0.792 & $1.330(0.646-2.740)$ & 0.439 \\
\hline $\mathrm{HCT}$ & $0.726(0.417-1.264)$ & 0.258 & $1.164(0.568-2.386)$ & 0.678 \\
\hline $\mathrm{MCV}$ & $1.585(0.907-2.770)$ & 0.106 & $1.644(0.792-3.416)$ & 0.182 \\
\hline $\mathrm{MCH}$ & $1.959(1.114-3.445)$ & 0.020 & $1.848(0.885-3.858)$ & 0.102 \\
\hline $\mathrm{MCHC}$ & $1.108(0.640-1.920)$ & 0.714 & $1.015(0.495-2.082)$ & 0.967 \\
\hline NLR & $1.808(1.021-3.202)$ & 0.042 & $1.230(0.591-2.559)$ & 0.579 \\
\hline
\end{tabular}

CI, confidence interval; ER, estrogen receptor; PR, progesterone receptor; HER2, human epidermal growth factor receptor 2; RBC, red blood cell; $\mathrm{HGB}$, hemoglobin; $\mathrm{HCT}$, hematocrit; MCV, mean corpuscular volume; $\mathrm{MCH}$, mean corpuscular hemoglobin; MCHC, mean corpuscular hemoglobin concentration; NLR, neutrophil-to-lymphocyte ratio.
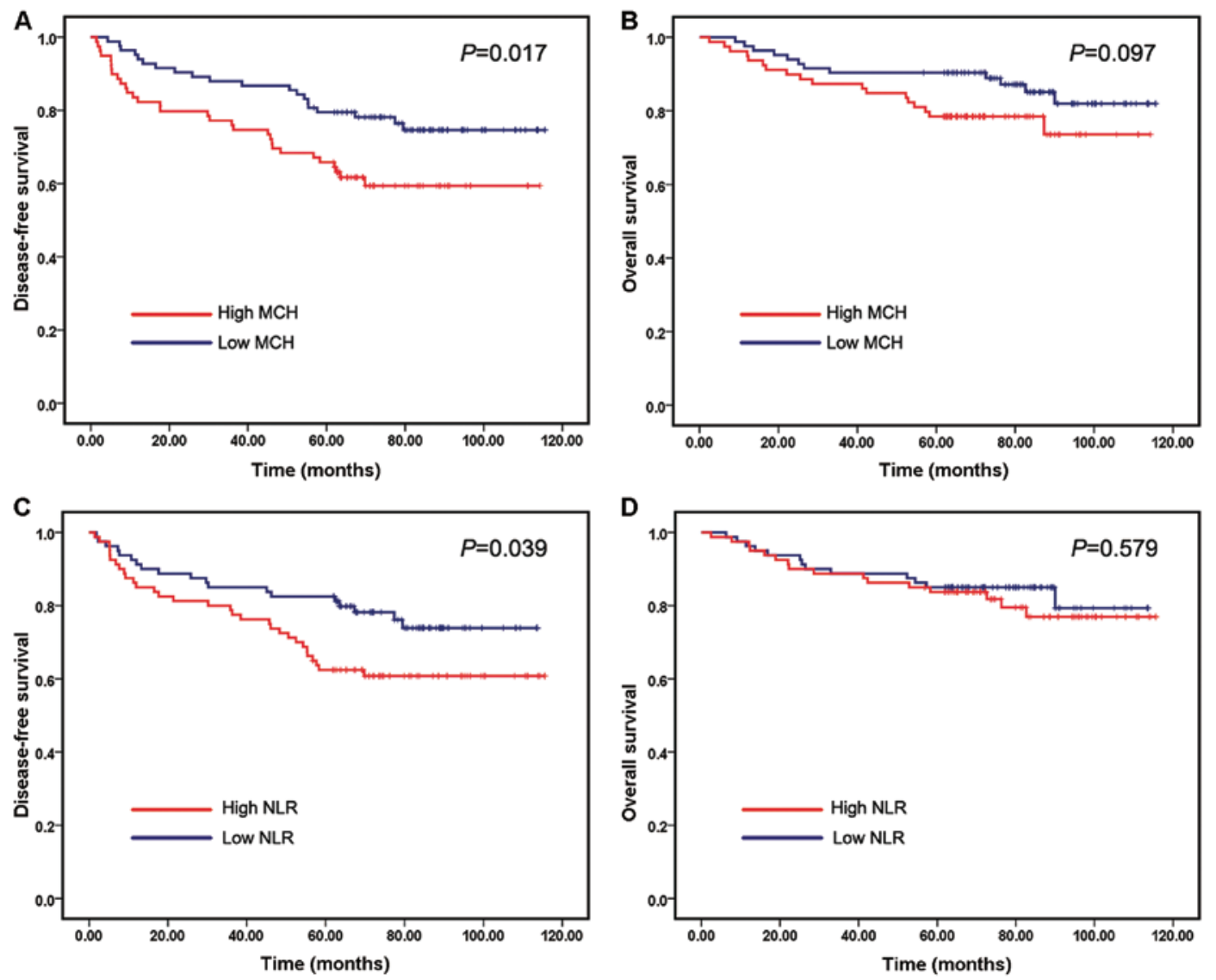

Figure 1. Prediction of DFS and OS in breast cancer patients according to the MCH and NLR. (A) Kaplan-Meier estimates of the DFS in patients in the high and low MCH groups. (B) Kaplan-Meier estimates of the OS in patients in the high and low MCH groups. (C) Kaplan-Meier estimates of the DFS in patients in the high and low NLR groups. (D) Kaplan-Meier estimates of the OS in patients in the high and low NLR groups. The marks on the graph lines represent censored samples. P-value refers to two-sided log-rank tests. DFS, disease-free survival; OS, overall survival; MCH, mean corpuscular hemoglobin; NLR, neutrophil-to-lymphocyte ratio. 
Table III. Multivariate model predicting DFS using MCH.

DFS

\begin{tabular}{lcc}
\cline { 2 - 3 } Factor & Hazard ratio (95\% CI) & P-value \\
\hline Tumor size, T2-T4 vs. T1 & $2.800(1.146-6.841)$ & 0.024 \\
Lymph node N1-3 vs. N0 & $1.525(0.625-3.725)$ & 0.354 \\
Histology grade, III vs. I/II & $1.405(0.776-2.544)$ & 0.261 \\
Clinical stage, III vs. I/II & $2.244(1.042-4.830)$ & 0.039 \\
MCH & $1.975(1.118-3.487)$ & 0.019
\end{tabular}

DFS, disease-free survival; MCH, mean corpuscular hemoglobin; CI, confidence interval.

Table IV. Multivariate model predicting DFS using NLR.

DFS

Factor

Hazard ratio (95\% CI) P-value

Tumor size, T2-T4 vs. T1

$2.503(1.023-6.126) \quad 0.044$

Lymph node N1-3 vs. N0

$1.453(0.596-3.541) \quad 0.411$

Histology grade, III vs. I/II

$1.440(0.792-2.618)$

0.232

Clinical stage, III vs. I/II

$2.253(1.041-4.876)$

0.039

NLR

$1.435(0.803-2.563)$

0.223

DFS, disease-free survival; NLR, neutrophil-to-lymphocyte ratio; CI, confidence interval.

any independent prognostic significance regarding OS and DFS.

Using median values as cut-off points, hematological parameters were stratified into high and low groups. Other than MCH and NLR, no parameters were found to be significantly correlated with DFS and OS in our cohort. As markers of anemia, HGB and MCV have been evaluated in a number of studies. Anemia is a frequent complication of cancer and cancer therapy, which has been shown to be highly associated with patient energy levels and quality of life scores, but also to exert a prognostic effect in several types of cancer, including breast cancer $(17,24)$. From the European Cancer Anemia Survey prospective study in $2001,62 \%$ of the 3,278 patients with breast cancer developed anemia at least once during the study follow-up period (25). A number of investigators have suggested an association between HGB level during chemotherapy and local relapse-free survival in predicting breast cancer outcome $(17,21)$. However, HGB did not exhibit significant prognostic value in this study. Previous studies have reported the effect of MCV increase on prognosis in patients with metastatic breast cancer treated with capecitabine $(9,22)$. In this study, MCV only exhibited a trend of higher mortality in patients (data not shown). A possible explanation is that the test parameters were recorded prior to treatment in our study, compared with those collected during the treatment process in those studies.
Clinical stage, tumor size and ER status are known predictors of prognosis in the patients with breast cancer. However, OS and DFS may differ widely in patients with the same status who receive the same treatment, suggesting that other, as yet undetermined, factors may affect prognosis. As regards pretreatment hematological parameters that have been predictive of patient prognosis, we next performed univariate and multivariate analyses of factors predictive of DFS and OS in patients with breast cancer. We found that clinical stage, tumor size, histological grade and pretreatment $\mathrm{MCH}$ were prognostic factors for DFS in our patient cohort. $\mathrm{MCH}$ represents the absolute amount of hemoglobin in the average red cell in a sample. Since the correlation between $\mathrm{MCH}$ and tumors has not been extensively investigated, the mechanisms underlying the association of high $\mathrm{MCH}$ with poor DFS in breast cancer patients is poorly understood. One potential mechanism underlying the prognostic impact of $\mathrm{MCH}$ may be the association of elevated $\mathrm{MCH}$ with metabolism. Tarocco et al (26) reported that elevated $\mathrm{MCH}$ was found to be negatively correlated with low level of serum folic acid and vitamin B12, which are important cofactors in DNA synthesis, repair and methylation $(27,28)$. Deficient folate and vitamin B12 levels may affect the prognosis of breast cancer patients by reducing the availability of S-adenosylmethionine for DNA methylation (29). A population-based case-control study of breast cancer conducted in urban Shanghai during 1996-1998 (28) reported evidence of a decreased risk of breast cancer associated with high consumption of folate. Several reports also revealed an association between higher plasma levels of folate and reduced risk of developing breast cancer (30-32).

A number of recent studies have suggested that an elevated NLR is associated with poor survival of patients with cancer (33). The mechanisms underlying the association of high NLR with poor outcome of cancer patients are poorly understood, but may be explained by the following facts: An association with inflammation may be underlying the prognostic impact of NLR (7). The contribution of host inflammatory reactions to cancer development has been reported. Immunocompetent lymphocytes and neutrophils may play an important role in the systemic inflammatory response (34). Azab et al (10) first evaluated NLR in predicting mortality in breast cancer patients and found that NLR was an independent predictor of mortality in breast cancer patients with NLR >3.3. Noh et al reported that patients with an elevated pretreatment NLR exhibited poorer DFS compared with patients without elevated NLR, which was most evident in the luminal A subtype (14). Similar results were reported in several other studies on breast cancer $(11,13,15,16)$. In this study, the breast cancer patients with elevated NLR exhibited shorter DFS times compared with those without elevated NLR ( $\mathrm{P}=0.039$; Fig. 1C). This was consistent with previously reported results $(11,14)$, while there was no significant trend for elevated NLR to be associated with worse patient OS, and NLR was not found to be an independent predictor of DFS or OS in breast cancer patients. The possible reasons for this may be as follows: First, the median value of NLR was used as a cut-off point to stratify high and low NLR groups, which is different from other reports; and second, our small sample size may reflect a selection bias to some extent. 
There were certain limitations and potential biases to this study. First, only Chinese breast cancer patients were included in our study. Due to small sample size, larger scale and multicenter studies should be performed to elucidate the accuracy and clinical value of $\mathrm{MCH}$. Second, due to its retrospective nature, some records of clinical details, such as body mass index, carbohydrate antigen 15-3 and folic acid concentration, were not available in this study. Thus, the correlation between $\mathrm{MCH}$ and these parameters remains unclear. Furthermore, we were unable to determine whether preoperative $\mathrm{MCH}$ was a better predictor of DFS compared with conventional prognostic factors.

In conclusion, we demonstrated that patients with high pretreatment $\mathrm{MCH}$ may be predicted to exhibit shorter DFS time compared with those with low pretreatment $\mathrm{MCH}$. $\mathrm{MCH}$ is an independent predictor associated with DFS. Additional studies, however, are required for further validation in a larger population from different races and regions.

\section{Acknowledgements}

The present study was supported by grants from the National Natural Science Foundation of China (nos. 81472497, 81572620 and 31100960) and the Shandong Provincial Natural Science Foundation of China (no. ZR2015HM003).

\section{References}

1. Eichelser C, Flesch-Janys D, Chang-Claude J, Pantel K and Schwarzenbach H: Deregulated serum concentrations of circulating cell-free microRNAs miR-17, miR-34a, miR-155, and miR-373 in human breast cancer development and progression. Clin Chem 59: 1489-1496, 2013.

2. Wei C, Cao Y, Yang X, Zheng Z, Guan K, Wang Q, Tai Y, Zhang Y, Ma S, Cao Y, et al: Elevated expression of TANK-binding kinase 1 enhances tamoxifen resistance in breast cancer. Proc Natl Acad Sci USA 111: E601-E610, 2014.

3. Cancer Genome Atlas Network: Comprehensive molecular portraits of human breast tumours. Nature 490: 61-70, 2012.

4. Fan L, Strasser-Weippl K, Li JJ, St Louis J, Finkelstein DM, Yu KD, Chen WQ, Shao ZM and Goss PE: Breast cancer in China. Lancet Oncol 15: e279-e289, 2014.

5. Li J, Zhang BN, Fan JH, Pang Y, Zhang P, Wang SL, Zheng S, Zhang B, Yang HJ, Xie XM, et al: A nation-wide multicenter 10-year (1999-2008) retrospective clinical epidemiological study of female breast cancer in China. BMC cancer 11: 364, 2011.

6. DeSantis C, Ma J, Bryan L and Jemal A: Breast cancer statistics, 2013. CA Cancer J Clin 64: 52-62, 2014.

7. Loi S, Sirtaine N, Piette F, Salgado R, Viale G, Van Eenoo F, Rouas G, Francis P, Crown JP, Hitre E, et al: Prognostic and predictive value of tumor-infiltrating lymphocytes in a phase III randomized adjuvant breast cancer trial in node-positive breast cancer comparing the addition of docetaxel to doxorubicin with doxorubicin-based chemotherapy: BIG 02-98. J Clin Oncol 31: 860-867, 2013

8. Molina R, Auge JM, Farrus B, Zanón G, Pahisa J, Muñoz M, Torne A, Filella X, Escudero JM, Fernandez P and Velasco M: Prospective evaluation of carcinoembryonic antigen (CEA) and carbohydrate antigen 15.3 (CA 15.3) in patients with primary locoregional breast cancer. Clin Chem 56: 1148-1157, 2010.

9. Arslan C, Aksoy S, Dizdar O, Kurt M, Güler N, Ozisik Y, Güllü I and Altundag K: Increased mean corpuscular volume of erythrocytes during capecitabine treatment: A simple surrogate marker for clinical response. Tumori 97: 711-716, 2011.

10. Azab B, Bhatt VR, Phookan J, Murukutla S, Kohn N, Terjanian T and Widmann WD: Usefulness of the neutrophil-to-lymphocyte ratio in predicting short- and long-term mortality in breast cancer patients. Ann Surg Oncol 19: 217-224, 2012.

11. Nakano K, Hosoda $M$, Yamamoto $M$ and Yamashita $H$ : Prognostic significance of pre-treatment neutrophil: Lymphocyte ratio in Japanese patients with breast cancer. Anticancer Res 34: $3819-3824,2014$
12. Ishizuka $M$, Nagata $H$, Takagi $K$, Iwasaki $Y$ and Kubota $K$ : Combination of platelet count and neutrophil to lymphocyte ratio is a useful predictor of postoperative survival in patients with colorectal cancer. Br J Cancer 109: 401-407, 2013.

13. Dirican A, Kucukzeybek BB, Alacacioglu A, Kucukzeybek Y, Erten C, Varol U, Somali I, Demir L, Bayoglu IV and Yildiz Y, et al: Do the derived neutrophil to lymphocyte ratio and the neutrophil to lymphocyte ratio predict prognosis in breast cancer? Int J Clin Oncol 20: 70-81, 2015.

14. Noh H, Eomm M and Han A: Usefulness of pretreatment neutrophil to lymphocyte ratio in predicting disease-specific survival in breast cancer patients. J Breast Cancer 16: 55-59, 2013.

15. Azab B, Shah N, Radbel J, Tan P, Bhatt V, Vonfrolio S, Habeshy A, Picon A and Bloom S: Pretreatment neutrophil/lymphocyte ratio is superior to platelet/lymphocyte ratio as a predictor of long-term mortality in breast cancer patients. Med Oncol 30: 432, 2013.

16. Koh YW, Lee HJ, Ahn JH, Lee JW and Gong G: Prognostic significance of the ratio of absolute neutrophil to lymphocyte counts for breast cancer patients with ER/PR-positivity and HER2-negativity in neoadjuvant setting. Tumour Biol 35: 9823-9830, 2014.

17. Dubsky P, Sevelda P, Jakesz R, Hausmaninger H, Samonigg H, Seifert M, Denison U, Mlineritsch B, Steger G, Kwasny W, et al: Anemia is a significant prognostic factor in local relapse-free survival of premenopausal primary breast cancer patients receiving adjuvant cyclophosphamide/methotrexate/5-fluorouracil chemotherapy. Clin Cancer Res 14: 2082-2087, 2008.

18. Bottini A, Berruti A, Brizzi MP, Bersiga A, Generali D, Allevi G, Aguggini S, Bolsi G, Bonardi S, Bertoli G, et al: Pretreatment haemoglobin levels significantly predict the tumour response to primary chemotherapy in human breast cancer. Br J Cancer 89: 977-982, 2003.

19. Vaupel P, Mayer A, Briest S and Höckel M: Hypoxia in breast cancer: Role of blood flow, oxygen diffusion distances, and anemia in the development of oxygen depletion. Adv Exp Med Biol 566: 333-342, 2005.

20. Peters-Engl C, Cassik P, Schmidt I, Denison U, Medl M, Pokieser W and Sevelda P: Impact of haemoglobin levels during adjuvant chemotherapy on the survival of patients with primary breast cancer. Acta Oncol 44: 129-133, 2005.

21. Boehm DU, Lebrecht A, Schmidt M, Siggelkow W, Lindner C, Litz A, Ulbrich E and Koelbl H: Prognostic impact of haemoglobin levels in breast cancer. Anticancer Res 27: 1223-1226, 2007.

22. Karvellas CJ, Sawyer M, Hamilton M and Mackey JR: Effect of capecitabine on mean corpuscular volume in patients with metastatic breast cancer. Am J Clin Oncol 27: 364-368, 2004.

23. Bozkurt O, Berk V, Kaplan MA, Cetin B, Ozaslan E, Karaca H, Inanc M, Duran AO and Ozkan M: Lack of prognostic value of mean corpuscular volume with capecitabine therapy in metastatic breast cancer. Asian Pac J Cancer Prev 15: 2501-2504, 2014.

24. Ludwig H, Müldür E, Endler G and Hübl W: Prevalence of iron deficiency across different tumors and its association with poor performance status, disease status and anemia. Ann Oncol 24: 1886-1892, 2013.

25. Ludwig H, Van Belle S, Barrett-Lee P, Birgegård G, Bokemeyer C, Gascón P, Kosmidis P, Krzakowski M, Nortier J, Olmi P, et al: The European Cancer Anaemia Survey (ECAS): A large, multinational, prospective survey defining the prevalence, incidence, and treatment of anaemia in cancer patients. Eur J Cancer 40: 2293-2306, 2004.

26. Tarocco RP, Faro G, Sargiotto A and Ansermin A: Folate and vitamin B12 deficiency. Characterization of parameters for early diagnosis. Recenti Prog Med 80: 547-550, 1989 (In Italian).

27. Toprak B, Yalcin HZ and Colak A: Vitamin B12 and folate deficiency: Should we use a different cutoff value for hematologic disorders? Int J Lab Hematol 36: 409-414, 2014.

28. Shrubsole MJ, Jin F, Dai Q, Shu XO, Potter JD, Hebert JR, Gao YT and Zheng W: Dietary folate intake and breast cancer risk: Results from the Shanghai Breast Cancer Study. Cancer Res 61: 7136-7141, 2001.

29. Blount BC, Mack MM, Wehr CM, MacGregor JT, Hiatt RA, Wang G, Wickramasinghe SN, Everson RB and Ames BN: Folate deficiency causes uracil misincorporation into human DNA and chromosome breakage: Implications for cancer and neuronal damage. Proc Natl Acad Sci USA 94: 3290-3295, 1997.

30. Zhang SM, Willett WC, Selhub J, Hunter DJ, Giovannucci EL, Holmes MD, Colditz GA and Hankinson SE: Plasma folate, vitamin B6, vitamin B12, homocysteine, and risk of breast cancer. J Natl Cancer Inst 95: 373-380, 2003. 
31. Lin J, Lee IM, Cook NR, Selhub J, Manson JE, Buring JE and Zhang SM: Plasma folate, vitamin B-6, vitamin B-12, and risk of breast cancer in women. Am J Clin Nutr 87: 734-743, 2008.

32. Li B, Lu Y, Wang L and Zhang CX: Folate intake and breast cancer prognosis: A meta-analysis of prospective observational studies. Eur J Cancer Prev 24: 113-121, 2015.

33. Templeton AJ, McNamara MG, Seruga B, Vera-Badillo FE, Aneja P, Ocaña A, Leibowitz-Amit R, Sonpavde G, Knox JJ, Tran B, et al: Prognostic role of neutrophil-to-lymphocyte ratio in solid tumors: A systematic review and meta-analysis. J Natl Cancer Inst 106: dju124, 2014.
34. Inanc M, Duran AO, Karaca H, Berk V, Bozkurt O, Ozaslan E and Ozkan M: Haematologic parameters in metastatic colorectal cancer patients treated with capecitabine combination therapy. Asian Pac J Cancer Prev 15: 253-256, 2014. 